\title{
Robust Filtering for Bilinear Uncertain Stochastic Discrete-Time Systems
}

\author{
Zidong Wang, Member, IEEE, and Hong Qiao, Member, IEEE
}

\begin{abstract}
This paper deals with the robust filtering problem for uncertain bilinear stochastic discrete-time systems with estimation error variance constraints. The uncertainties are allowed to be norm-bounded and enter into both the state and measurement matrices. We focus on the design of linear filters, such that for all admissible parameter uncertainties, the error state of the bilinear stochastic system is mean square bounded, and the steadystate variance of the estimation error of each state is not more than the individual prespecified value. It is shown that the design of the robust filters can be carried out by solving some algebraic quadratic matrix inequalities. In particular, we establish both the existence conditions and the explicit expression of desired robust filters. A numerical example is included to show the applicability of the present method.
\end{abstract}

Index Terms-Bilinear stochastic systems, discrete-time systems, quadratic matrix inequalities, robust filtering, uncertain systems.

\section{INTRODUCTION}

$\mathbf{K}$ ALMAN filtering is one of the celebrated $\mathrm{H}_{2}$ filtering approaches that is widely used in various fields of signal processing and control; see [2]. This filtering approach assumes that the system under consideration has known dynamics described by certain well-posed model, and its disturbances are Gaussian noises with known statistics. These assumptions limit the application scope of the Kalman filtering technique when there are uncertainties in either the exogenous input signals or the system model. It has been known that the standard Kalman filtering algorithms will generally not guarantee satisfactory performance when there exists uncertainty in the system model [2]. This has led to the recent development of alternative design methods for $H_{\infty}$ filters and robust filters.

For uncertain stochastic systems, it is reasonable to evaluate the filter performance in terms of mean square error and strive for a suitable robustification of the classical Kalman filter. Therefore, the study of the so-called cost guaranteed filters that minimize an easy-to-compute upper bound on the worst performance has recently gained growing interest, and many papers have been published in this area; see [11], [15] and the

Manuscript received October 12, 2000; revised October 29, 2001. This work was supported in part by the City University of Hong Kong, the University of Kaiserslautern, Germany, and the Alexander von Humboldt Foundation, Germany. The associate editor coordinating the review of this paper and approving it for publication was Dr. Gonzalo R. Arce.

Z. Wang is with the Control Theory and Applications Centre, School of Mathematical and Information Sciences, Coventry University, Coventry, U.K. (e-mail: Zidong.Wang@coventry.ac.uk).

H. Qiao was with the Department of Manufacturing Engineering and Engineering Management, City University of Hong Kong, Kowloon, Hong Kong. She is now with the Computation Department, University of Manchester Institute of Science and Technology, Manchester, U.K.

Publisher Item Identifier S 1053-587X(02)01343-0. references therein. A common feature of these papers is that they have focused on designing a filter that first provides an upper bound on the variance of the estimation error for all admissible parameter perturbations and then minimizes this bound. It is remarkable that in this case, the associated upper bound is not specified a priori, and the resulting optimal robust filters are often unique in certain cases.

On the other hand, it is quite common in filtering problems to have performance objectives that are expressed as upper bounds on the variances of the estimation error. For example, in the problem of tracking maneuvering target, it is often desired to obtain the filter gain such that the estimation value of system state is situated in the prespecified effective region. Clearly, this performance requirement can be described as upper bounds on the estimation error variances of the states; see e.g., [19] and [30]. In this case, the steady-state error variance is not required to be minimal but should not be more than a prescribed upper bound. Note that it is usually difficult to utilize traditional methods to deal with this class of variance-constrained filtering problems. For instance, the theory of weighted least-squares estimation [2] minimizes a weighted scalar sum of the error variances of the state estimation, but minimizing a scalar sum does not ensure that the multiple variance requirements will be satisfied.

Fortunately, the error covariance assignment (ECA) theory developed in [30] provides an alternative and more straightforward methodology for designing filter gains that satisfy the above performance objectives. This methodology could provide a closed-form solution for directly assigning the specified steady-state estimation error covariance. Subsequently, the ECA theory has been extended to the sampled-data systems in [24], to the bilinear systems in [22], and to the parameter uncertain systems in [23], [25], and [26], where upper bound constraints are imposed on the steady-state estimation error variance. Unlike scalar objective designs, which often lead to unique solutions, such as linear quadratic techniques in which only one gain matrix minimizes a scalar cost function, the variance-constrained filter designs often lead to nonunique solutions since the addressed problems are multiobjective design tasks. As can be seen in the ECA theory, the set of desired variance-constrained filters is usually large. Therefore, after assigning to the estimation error a specified variance upper bound, there exists much freedom that may be used to attempt to achieve other desired performance requirements, such as robustness.

Among many practical systems, plants may be modeled by bilinear systems (BLSs) since some characteristics of nonlinear systems can be closely approximated by bilinear models rather than linearized models. The bilinear system is a kind of "nearly 
linear" yet nonlinear system. The related problems of bilinear systems, such as the state analysis and the parameter estimation, are much more difficult to solve than those of the linear systems. Up to now, it has been known that BLSs could describe many real processes in the fields of socioeconomics, ecology, agriculture, biology, and industry, etc. (see, e.g., [5], [6], [13], [16], [17], [20], [21], [28], and [29]). In particular, bilinear models are widely used to model nonlinear processes in signal and image processing, as well as communication systems analysis, such as channel equalization, echo cancellation, nonlinear tracking, electroencephalogram (EEG) signal classification, multiplicative disturbance tracking, etc.; see [13] and references therein.

The observer design problem has been intensively studied in [29] for discrete-time stochastic bilinear systems (also called "state-dependent noise systems" because the structured parameter perturbations on the system matrix are modeled as zero mean white noises; see [4], [20], [28], and [29]). In [7], a finitedimensional linear filter has been proposed for a class of continuous-time bilinear stochastic systems, which could provide suboptimal state estimate instead of the conditional statistics. The control problems for bilinear stochastic continuous- and discrete-time systems with state covariance assignment have been investigated in [28] and [20], respectively. Recently, in [22], the state estimation problem has been tackled for discrete-time bilinear stochastic systems with error covariance placement. Necessary and sufficient conditions have been derived for the existence of the desired estimators, and the analytical expression of these estimators has been provided in [22]. However, so far, the issue of variance-constrained filtering for bilinear uncertain stochastic systems has not been fully investigated and remains important.

This paper is concerned with the design of robust filters for bilinear uncertain stochastic discrete-time systems subjected to the upper bound constraints on the estimation error variance. The purpose of the problem addressed is to design the filters for the bilinear uncertain stochastic discrete-time systems such that the steady-state estimation error variances are less than the prespecified upper bounds. A simple, effective matrix inequality approach is developed to solve this problem. Specifically, a set of the upper bounds on estimation error covariance that certain bilinear error dynamic processes may obey are first presented, all filters that assign these upper bounds to the estimation error variances are then explicitly characterized, and finally, the solvability of the assignability conditions is discussed. An illustrative example is used to demonstrate the effectiveness of the proposed design procedure.

The remainder of this paper is organized as follows. In Section II, the robust Kalman filtering problem for discrete bilinear stochastic systems subject to norm-bounded parameter uncertainty is formulated. An algorithm for the filter design is developed in Section III, which guarantees the prespecified upper bound on the steady-state state estimation error variance. An illustrative example is given in Section IV, and some concluding remarks are drawn in Section V.

Notation: The notations in this paper are quite standard. $\mathbb{R}^{n}$ and $\mathbb{R}^{n \times m}$ denote, respectively, the $n$ dimensional Euclidean space and the set of all $n \times m$ real matrices. The superscript " $T$ " denotes the transpose, and the notation $X \geq Y$ (respec- tively, $X>Y$ ), where $X$ and $Y$ are symmetric matrices, means that $X-Y$ is positive semi-definite (respectively, positive definite). $I$ is the identity matrix with compatible dimension. Let $\left(\Omega, \mathcal{F},\left\{\mathcal{F}_{t}\right\}_{t>0}, P\right)$ be a complete probability space with a filtration $\left\{\mathcal{F}_{t}\right\}_{t \geq 0}$ satisfying the usual conditions (i.e., the filtration contains all $P$-null sets and is right continuous). $\mathcal{E}\{\cdot\}$ stands for the mathematical expectation operator with respect to the given probability measure $P$. Sometimes, the arguments of a function will be omitted in the analysis when no confusion can arise.

\section{PROBlem Formulation AND PRELIMINARY RESUltS}

Consider the following bilinear uncertain discrete-time stochastic system.

$$
x(k+1)=(A+\Delta A) x(k)+\sum_{i=1}^{m} H_{i} x(k) v_{i}(k)+w(k)
$$

and the measurement equation

$$
y(k)=(C+\Delta C) x(k)+z(k)
$$

where $x(k) \in \mathbb{R}^{n}$ is the state, $y(k) \in \mathbb{R}^{p}$ is the measurement output, and $w(k) \in \mathbb{R}^{n}$ and $z(k) \in \mathbb{R}^{p}$ are uncorrelated stationary zero mean white noise sequences with respective covariance $W \geq 0$ and $Z>0$. The stochastic sequence

$$
v(k):=\left[\begin{array}{llll}
v_{1}(k) & v_{2}(k) & \cdots & v_{m}(k)
\end{array}\right]^{T} \in \mathbb{R}^{m}
$$

is a vector satisfying

$$
\mathcal{E} v(k)=0, \quad \mathcal{E} v(l) v^{T}(j)= \begin{cases}0, & \text { if } l \neq j \\ I, & \text { if } l=j\end{cases}
$$

for $l, j=1,2, \ldots, m$, where $\mathcal{E}\{\cdot\}$ is the mathematical expectation operator. The initial state $x(0)=x_{0}$ is a random vector that is independent of $w(k)$ and $z(k) . A, H_{i}(i=1,2, \ldots, m)$ and $C$ are known constant matrices with appropriate dimensions.

The matrices $\triangle A$ and $\Delta C$, which may be time-varying, represent the norm-bounded parameter uncertainties and satisfy

$$
\left[\begin{array}{l}
\Delta A \\
\Delta C
\end{array}\right]=\left[\begin{array}{l}
M_{1} \\
M_{2}
\end{array}\right] F N
$$

where $F \in \mathbb{R}^{i \times j}$ is a real uncertain matrix with Lebesgue measurable elements and meets

$$
F^{T} F \leq I
$$

and $M_{1}, M_{2}, N$ are known real constant matrices of appropriate dimensions that specify how the uncertain parameters in $F$ enter the nominal matrices $A$ and $C$. The uncertainties $\Delta A, \Delta C$ are said to be admissible if both (3) and (4) are satisfied.

Remark 1: The kind of bilinear stochastic discrete-time systems formulated by (1) and (2) without uncertainties has been extensively studied in many papers (see, e.g., [4], [20], [29], and the references therein) and is sometimes called the "state-dependent noise system." It can be noticed that there are two kinds of "uncertainties" posing on the nominal matrix $A$, 
namely, the deterministic uncertainty $\Delta A$ that can be regarded as the additive energy-bounded noise and the stochastic perturbation $\sum_{i=1}^{m} H_{i} v_{i}(k)$ that is the multiplicative noise with known statistics. Both kinds of uncertainties have been well investigated in the literature.

Remark 2: The parameter uncertainty structure as in (3) and (4) has been widely used in the problems of robust control and robust filtering of uncertain systems (see, e.g., [26]). Many practical systems possess parameter uncertainties that can be either exactly modeled or overbounded by (4). The unknown matrix $F(t)$ in (3) can be time varying and can even be state dependent, i.e., $F:=F(t, x(t))$, as long as (4) is satisfied.

Throughout this paper, we will need the following assumption.

Assumption 1: The matrix $A$ is Schur stable (i.e., all eigenvalues of $A$ are located inside the unit circle in the complex plane) and are nonsingular.

In this paper, we adopt the following linear full-order filter

$$
\hat{x}(k+1)=G \hat{x}(k)+K y(k)
$$

where $\hat{x}(k)$ stands for the state estimate, and $G$ and $K$ are filter parameters to be designed.

Define the estimation error and the estimation error covariance, respectively, as follows.

$$
\begin{aligned}
e(k) & :=x(k)-\hat{x}(k), \\
P(k) & :=\mathcal{E}\left[e(k) e^{T}(k)\right] .
\end{aligned}
$$

Then, it follows from (1) and (2) and from (5)-(7) that

$$
\begin{aligned}
e(k+1)=G e(k)+ & {\left[(A-G-K C)+\sum_{i=1}^{m} H_{i}\left(v_{i}(k) I\right)+\Delta A\right.} \\
& -K(\Delta C)] \cdot x(k)+w(k)-K z(k) . \quad(8)
\end{aligned}
$$

Define

$$
\begin{aligned}
x_{f}(k) & :=\left[\begin{array}{l}
x(k) \\
e(k)
\end{array}\right] \\
A_{f} & :=\left[\begin{array}{cc}
A & 0 \\
A-G-K C & G
\end{array}\right] \\
M_{f} & :=\left[\begin{array}{c}
M_{1} \\
M_{1}-K M_{2}
\end{array}\right] \\
N_{f} & :=\left[\begin{array}{ll}
N & 0
\end{array}\right] \\
\Delta A_{f} & =M_{f} F N_{f} \\
H_{f} & :=\left[\begin{array}{ll}
\sum_{i=1}^{m} H_{i}\left(v_{i}(k) I\right) & 0 \\
\sum_{i=1}^{m} H_{i}\left(v_{i}(k) I\right) & 0
\end{array}\right] \\
J_{i} & :=\left[\begin{array}{ll}
H_{i} & 0 \\
H_{i} & 0
\end{array}\right]
\end{aligned}
$$

$$
\begin{aligned}
W_{f} & :=B_{f} B_{f}^{T} \\
& :=\left[\begin{array}{ll}
W & W \\
W & W+K Z K^{T}
\end{array}\right] \\
X(k) & :=\mathcal{E}\left[x_{f}(k) x_{f}^{T}(k)\right] \\
& :=\left[\begin{array}{cc}
X_{x x}(k) & X_{x e}(k) \\
X_{x e}^{T}(k) & X_{e e}(k)
\end{array}\right] .
\end{aligned}
$$

Considering (1) and (8), we obtain the following augmented system.

$$
\begin{aligned}
x_{f}(k+1) & =\left(A_{f}+\Delta A_{f}+H_{f}\right) x_{f}(k)+B_{f} w_{f}(k), \\
x_{f}(0) & =\left[\begin{array}{ll}
x_{0} & x_{0}-\hat{x}_{0}
\end{array}\right]^{T}
\end{aligned}
$$

where $w_{f}(k)$ stands for a zero mean Gaussian white noise sequence with covariance $I$.

Now, multiplying both sides of (18) by their transposes and taking the expectation, we have

$$
\begin{aligned}
X(k+1)=\left(A_{f}+\Delta A_{f}\right) X(k) & \left(A_{f}+\Delta A_{f}\right)^{T} \\
& +\sum_{i=1}^{m} J_{i} X(k) J_{i}^{T}+W_{f}
\end{aligned}
$$

where $J_{i}, W_{f}$, and $X(k)$ are defined in (15)-(17), respectively.

We know from [1], [8], and [9] that if the state of the system (18) is mean square bounded (for the definition of mean square boundedness, see [1], [8], and [9]), the steady-state covariance $X$ of the system (18) defined by

$$
X:=\lim _{k \rightarrow \infty} X(k)
$$

exists and satisfies the following discrete-time modified Lyapunov equation.

$$
X=\left(A_{f}+\Delta A_{f}\right) X\left(A_{f}+\Delta A_{f}\right)^{T}+\sum_{i=1}^{m} J_{i} X J_{i}^{T}+W_{f} .
$$

Remark 3: It is necessary to discuss the conditions for the existence of the solution to (20). It follows from [1] that there exists a unique symmetric positive semi-definite solution to (20) if and only if

$$
\rho\left\{\left(A_{f}+\Delta A_{f}\right) \otimes\left(A_{f}+\Delta A_{f}\right)+\sum_{i=1}^{m} J_{i} \otimes J_{i}\right\}<1
$$

where $\rho$ is the spectral radius, and $\otimes$ is the Kronecker product. Furthermore, we know from [1] that (21) is equivalent to the mean square boundedness of the state of the system (18), and hence, (21) will guarantee the convergence of $X(k)$ in (19) to a constant value $X$.

Define

$$
X_{e e}:=\lim _{k \rightarrow \infty} \mathcal{E}\left[e(k) e^{T}(k)\right] .
$$

The purpose of this paper is to design the filter parameters $G$ and $K$ such that for all admissible perturbations $\Delta A$ and $\Delta C$, the following requirements are met simultaneously.

1) The state of the augmented system (18) is mean square bounded. 
2) The steady-state error covariance $X_{e e}$ meets

$$
\left[X_{e e}\right]_{i i} \leq \sigma_{i}^{2}, \quad i=1,2, \ldots, n
$$

where $\left[X_{e e}\right]_{i i}$ means the steady-state variance of the $i$ th error state, and $\sigma_{i}^{2}(i=1,2, \ldots, n)$ denotes the prespecified steady-state error estimation variance constraint on the $i$ th state.

\section{MAIN RESUlTS AND PROOFS}

Let us first recall the some intermediate results that are introduced in the sequel as lemmas.

Lemma 1 [27]: Let a positive scalar $\varepsilon>0$ and a positive definite matrix $Q_{f}>0$ be such that

$$
N_{f} Q_{f} N_{f}^{T}<\varepsilon I \text {. }
$$

Then, we have that

$$
\begin{aligned}
& \left(A_{f}+\Delta A_{f}\right) Q_{f}\left(A_{f}+\Delta A_{f}\right)^{T} \\
& \quad \leq A_{f}\left(Q_{f}^{-1}-\varepsilon^{-1} N_{f}^{T} N_{f}\right)^{-1} A_{f}^{T}+\varepsilon M_{f} M_{f}^{T}
\end{aligned}
$$

holds for all admissible perturbations $\Delta A$ and $\Delta C$.

Lemma 2 (Schur Complement): Given constant matrices $\Omega_{1}, \Omega_{2}, \Omega_{3}$, where $\Omega_{1}=\Omega_{1}^{T}$ and $0<\Omega_{2}=\Omega_{2}^{T}$, then

$$
\Omega_{1}+\Omega_{3}^{T} \Omega_{2}^{-1} \Omega_{3}<0
$$

if and only if

$$
\left[\begin{array}{rr}
\Omega_{1} & \Omega_{3}^{T} \\
\Omega_{3} & -\Omega_{2}
\end{array}\right]<0
$$

or equivalently

$$
\left[\begin{array}{rr}
-\Omega_{2} & \Omega_{3} \\
\Omega_{3}^{T} & \Omega_{1}
\end{array}\right]<0
$$

Lemma 3 (Matrix Inverse Lemma): Let $A, B, C$, and $D$ be given matrices of appropriate dimension with $A, D$, and $D^{-1}+$ $C A^{-1} B$ being invertible; then

$$
(A+B D C)^{-1}=A^{-1}-A^{-1} B\left(D^{-1}+C A^{-1} B\right)^{-1} C A^{-1}
$$

holds.

The following lemma can be easily proved.

Lemma 4: For a given negative definite matrix $\Pi<0$ ( $\Pi \in$ $\left.\mathbb{R}^{n \times n}\right)$, there always exists a matrix $L \in \mathbb{R}^{n \times p}(p \leq n)$ such that

$$
\Pi+L L^{T}<0 .
$$

For technical convenience, we define the following additional notation.

$$
\begin{aligned}
\Phi & :=\left(P_{1}^{-1}-\varepsilon^{-1} N^{T} N\right)^{-1} A^{T} \\
\hat{A} & :=A+\left(W+\sum_{i=1}^{m} H_{i} P_{1} H_{i}^{T}+\varepsilon M_{1} M_{1}^{T}\right) \Phi^{-1} \\
\hat{C} & :=C+\varepsilon M_{2} M_{1}^{T} \Phi^{-1} \\
\Gamma & :=\Phi^{-1}\left(P_{1}^{-1}-\varepsilon^{-1} N^{T} N\right)^{-1}\left(\Phi^{-1}\right)^{T}
\end{aligned}
$$

$$
\begin{aligned}
R:= & Z+\varepsilon M_{2} M_{2}^{T}+\varepsilon^{2} M_{2} M_{1}^{T} \Gamma M_{1} M_{2}^{T}+\hat{C} P_{2} \hat{C}^{T} \\
\Theta:= & \hat{A} P_{2} \hat{C}^{T}+\varepsilon M_{1} M_{2}^{T} \\
& +\varepsilon\left(W+\sum_{i=1}^{m} H_{i} P_{1} H_{i}^{T}+\varepsilon M_{1} M_{1}^{T}\right) \Gamma M_{1} M_{2}^{T} \\
\Pi:= & \hat{A} P_{2} \hat{A}^{T}-P_{2}-\Theta R^{-1} \Theta^{T} \\
& +\left(W+\sum_{i=1}^{m} H_{i} P_{1} H_{i}^{T}+\varepsilon M_{1} M_{1}^{T}\right) \Gamma \\
& \cdot\left(W+\sum_{i=1}^{m} H_{i} P_{1} H_{i}^{T}+\varepsilon M_{1} M_{1}^{T}\right) \\
& +W+\sum_{i=1}^{m} H_{i} P_{1} H_{i}^{T}+\varepsilon M_{1} M_{1}^{T} .
\end{aligned}
$$

Now, we are in a position to establish our main results in this paper.

Theorem 1: Assume that there exists a positive scalar $\varepsilon$ such that the following two quadratic matrix inequalities

$$
\begin{aligned}
& A P_{1} A^{T}-P_{1}+A P_{1} N^{T}\left(\varepsilon I-N P_{1} N^{T}\right)^{-1} N P_{1} A^{T} \\
& +\varepsilon M_{1} M_{1}^{T}+W+\sum_{i=1}^{m} H_{i} P_{1} H_{i}^{T}<0
\end{aligned}
$$

$$
\Pi<0
$$

respectively, have positive definite solutions $P_{1}$ and $P_{2}$, where $P_{1}$ satisfies

$$
N P_{1} N^{T}<\varepsilon I .
$$

Moreover, let $L \in \mathbb{R}^{n \times p}(p \leq n)$ be an arbitrary matrix satisfying $\Pi+L L^{T}<0$ (see Lemma 4), and let $U \in \mathbb{R}^{p \times p}$ be an arbitrary orthogonal matrix (i.e., $U U^{T}=I$ ). Then, the filter (5) with the parameters determined by

$$
\begin{aligned}
K & =\Theta R^{-1}+L U R^{-1 / 2} \\
G & =\hat{A}-K \hat{C}
\end{aligned}
$$

will be such that for all admissible perturbations $\Delta A$ and $\Delta C$, we have the following.

1) The state of the augmented system (18) is mean square bounded.

2) The steady-state error covariance $X_{e e}$ satisfies

$$
X_{e e}<P_{2} \text {. }
$$

Proof: Since $A$ is assumed to be nonsingular, $\Phi^{-1}$ exists, and the definitions (24)-(30) are meaningful. We set

$$
P_{f}:=\left[\begin{array}{cc}
P_{1} & 0 \\
0 & P_{2}
\end{array}\right]
$$


Then, by means of Lemma 1 and the definitions (24)-(30), it is easily verified that

$$
\begin{aligned}
\left(A_{f}\right. & \left.+\Delta A_{f}\right) P_{f}\left(A_{f}+\Delta A_{f}\right)^{T}-P_{f}+\sum_{i=1}^{m} J_{i} P_{f} J_{i}^{T}+W_{f} \\
\leq & A_{f}\left(P_{f}^{-1}-\varepsilon^{-1} N_{f}^{T} N_{f}\right)^{-1} A_{f}^{T}+\varepsilon M_{f} M_{f}^{T}-P_{f} \\
& +\sum_{i=1}^{m} J_{i} P_{f} J_{i}^{T}+W_{f} \\
:= & \Psi:=\left[\begin{array}{ll}
\Psi_{11} & \Psi_{12} \\
\Psi_{12}^{T} & \Psi_{22}
\end{array}\right]
\end{aligned}
$$

where

$$
\begin{aligned}
\Psi_{11}= & A\left(P_{1}^{-1}-\varepsilon^{-1} N^{T} N\right)^{-1} A^{T}-P_{1}+\varepsilon M_{1} M_{1}^{T}+W \\
& +\sum_{i=1}^{m} H_{i} P_{1} H_{i}^{T} \\
\Psi_{12}= & A\left(P_{1}^{-1}-\varepsilon^{-1} N^{T} N\right)^{-1}(A-G-K C)^{T} \\
& +\varepsilon M_{1}\left(M_{1}-K M_{2}\right)^{T}+W+\sum_{i=1}^{m} H_{i} P_{1} H_{i}^{T} \\
\Psi_{22}= & G P_{2} G^{T}-P_{2}+(A-G-K C) \cdot\left(P_{1}^{-1}-\varepsilon^{-1} N^{T} N\right)^{-1} \\
& \cdot(A-G-K C)^{T}+\varepsilon\left(M_{1}-K M_{2}\right)\left(M_{1}-K M_{2}\right)^{T} \\
& +W+\sum_{i=1}^{m} H_{i} P_{1} H_{i}^{T}+K Z K^{T} .
\end{aligned}
$$

It follows immediately from the matrix inverse lemma (Lemma 3) that

$$
\left(P_{1}^{-1}-\varepsilon^{-1} N^{T} N\right)^{-1}=P_{1}+P_{1} N^{T}\left(\varepsilon I-N P_{1} N^{T}\right)^{-1} N P_{1}
$$

and then, the inequality (31) implies that

$$
\Psi_{11}<0 \text {. }
$$

To continue, by resorting to the definitions of $\hat{A}, \hat{C}$, , and the expression $G=\hat{A}-K \hat{C}$ in (34), we can rewrite (38) as

$$
\begin{aligned}
\Psi_{22}= & \hat{A} P_{2} \hat{A}^{T}-P_{2}+\left(W+\sum_{i=1}^{m} H_{i} P_{1} H_{i}^{T}+\varepsilon M_{1} M_{1}^{T}\right) \\
& \cdot \Gamma\left(W+\sum_{i=1}^{m} H_{i} P_{1} H_{i}^{T}+\varepsilon M_{1} M_{1}^{T}\right) \\
& +W+\sum_{i=1}^{m} H_{i} P_{1} H_{i}^{T}+\varepsilon M_{1} M_{1}^{T} \\
& -K \Theta^{T}-\Theta K^{T}+K R K^{T} \\
= & \hat{A} P_{2} \hat{A}^{T}-P_{2}+\left(W+\sum_{i=1}^{m} H_{i} P_{1} H_{i}^{T}+\varepsilon M_{1} M_{1}^{T}\right) \\
& \cdot \Gamma\left(W+\sum_{i=1}^{m} H_{i} P_{1} H_{i}^{T}+\varepsilon M_{1} M_{1}^{T}\right) \\
& +W+\sum_{i=1}^{m} H_{i} P_{1} H_{i}^{T}+\varepsilon M_{1} M_{1}^{T}-\Theta R^{-1} \Theta^{T} \\
& +\left(K R^{1 / 2}-\Theta R^{-1 / 2}\right)\left(K R^{1 / 2}-\Theta R^{-1 / 2}\right)^{T} \\
= & \Pi+\left(K R^{1 / 2}-\Theta R^{-1 / 2}\right)\left(K R^{1 / 2}-\Theta R^{-1 / 2}\right)^{T} .
\end{aligned}
$$

Noticing the expression of $K=\Theta R^{-1}+L U R^{-1 / 2}$ in (33) and the fact of $U U^{T}=I$, we obtain

$$
\left(K R^{1 / 2}-\Theta R^{-1 / 2}\right)\left(K R^{1 / 2}-\Theta R^{-1 / 2}\right)^{T}=L L^{T} .
$$

Thus, it follows from (39), the definition of the matrix $L$ ( $L \in$ $\mathbb{R}^{n \times p}$ ), and the inequality (32) that

$$
\Psi_{22}=\Pi+L L^{T}<0 .
$$

Moreover, substituting $G=\hat{A}-K \hat{C}$ into (37) immediately yields

$$
\Psi_{12}=0
$$

To this end, we arrive at the conclusion that $\Psi<0$. Therefore, it follows from (35) that

$$
\begin{aligned}
\left(A_{f}+\Delta A_{f}\right) P_{f}\left(A_{f}+\Delta A_{f}\right)^{T}-P_{f} & +\sum_{i=1}^{m} J_{i} P_{f} J_{i}^{T} \\
& =-W_{f}+\Psi<0
\end{aligned}
$$

which leads to (21). As discussed in Remark 3, we know that the state of the augmented system (18) is mean square bounded, and there exists a symmetric positive semi-definite solution to (20). This proves the first conclusion of this theorem.

Furthermore, subtract (20) from (40) to give

$$
\begin{aligned}
\left(A_{f}\right. & \left.+\Delta A_{f}\right)\left(P_{f}-X\right)\left(A_{f}+\Delta A_{f}\right)^{T}-\left(P_{f}-X\right) \\
& +\sum_{i=1}^{m} J_{i}\left(P_{f}-X\right) J_{i}^{T} \\
= & \Psi<0
\end{aligned}
$$

which indicates again from Remark 3 that

$$
P_{f}-X>0
$$

and therefore

$$
X_{e e}=[X]_{22}<\left[P_{f}\right]_{22}=P_{2} .
$$

This completes the proof of this theorem.

Remark 4: It is clear from Theorem 1 that if the quadratic matrix inequalities (31) and (32), respectively, have positive definite solutions $P_{1}, P_{2}\left(P_{2}\right.$ meets $\left.\left[P_{2}\right]_{i i} \leq \sigma_{i}^{2}, i=1,2, \cdots, n\right)$, then the filter (5) determined by (33) and (34) will be such that 1) the augmented system (18) is mean square bounded, and 2) $\left[X_{e e}\right]_{i i}<\left[P_{2}\right]_{i i} \leq \sigma_{i}^{2}, i=1,2, \cdots, n$. Hence, the design task of variance-constrained robust filtering for the uncertain bilinear systems will be accomplished. Note that the existence of a positive definite solution to (31) implies the asymptotical Schur stability of the system matrix $A$.

In practical applications, we can solve the quadratic matrix inequalities (QMIs) (31) and (32) subject to the constraints $\left[P_{2}\right]_{i i} \leq \sigma_{i}^{2}(i=1,2, \ldots, n)$ and then obtain the expected filter parameters immediately from (33)-(34). When we deal with the QMIs (31) and (32), the local numerical searching algorithms suggested in [3] and [12] are very effective for a relatively low-order model. The detailed discussion on the solving algorithms for QMIs can be found in [18].

For relatively high-order model, the aforementioned algorithms are no longer useful. Fortunately, the parameter $P_{2}$ of (32) is not included in (31). Therefore, if we could first solve (31) for $\varepsilon>0$ and $P_{1}>0$, then the inequality (32) is a standard Riccati-like matrix inequality for $P_{2}>0$ and could be solved by the generalized matrix Riccati inequality/equation approach 
(see [18]). We still need to focus on the algorithm for solving (31). By using the Schur Lemma (Lemma 2), we can convert (31) into the following linear matrix inequality (LMI).

$$
\left[\begin{array}{cc}
\Sigma & A P_{1} N^{T} \\
N P_{1} A^{T} & -\varepsilon I+N P_{1} N^{T}
\end{array}\right]<0
$$

where

$$
\Sigma:=A P_{1} A^{T}-P_{1}+\varepsilon M_{1} M_{1}^{T}+W+\sum_{i=1}^{m} H_{i} P_{1} H_{i}^{T} .
$$

The inequality (42), together with the inequality constraint

$$
-\varepsilon I+N P_{1} N^{T}<0
$$

are both linear on $\varepsilon>0$ and $P_{1}>0$. Therefore, we can employ the standard LMI techniques in [10] to check the solvability of the original matrix inequality (31).

It is worth mentioning that since LMIs intrinsically reflect constraints rather than optimality, they tend to offer more flexibility for combining several constraints. LMIs can now be solved efficiently via interior-point optimization algorithms, such as those described in [10]. Moreover, software like MATLAB LMI Toolbox are now available to solve such LMIs efficiently.

Remark 5: We point out that in the present design procedure of robust filters for bilinear systems, there exists much explicit freedom, such as the choices of the free parameters $L$ ( $L$ satisfies $\Pi+L L^{T}<0$ ), the orthogonal matrix $U$, etc. This remaining freedom provides the possibility for considering more performance constraints (e.g., the transient requirement and reliability behavior on the filtering process), which requires further investigations. Note that in [14], a similar freedom on an arbitrary orthogonal matrix in the parameterization of the set of filters was successfully employed to minimize the $H_{2}$ norm of the filtering error transfer function by solving an unconstrained parametric optimization problem over the set of filters.

\section{NuMERICAL EXAMPLE}

Consider a bilinear discrete-time uncertain stochastic system (1) and (2) with parameters as

$$
\begin{aligned}
A & =\left[\begin{array}{cc}
0.8 & 0.05 \\
-0.08 & -0.5
\end{array}\right], \quad C=\left[\begin{array}{ll}
1 & 0
\end{array}\right] \\
H_{1} & =\left[\begin{array}{cc}
0.01 & 0 \\
0 & 0.01
\end{array}\right], \quad H_{2}=\left[\begin{array}{cc}
0.02 & 0 \\
0 & 0.02
\end{array}\right] \\
M_{1} & =\left[\begin{array}{c}
0.08 \\
0.06
\end{array}\right], \quad M_{2}=0.1, \quad N=\left[\begin{array}{ll}
0.5 & 0.5
\end{array}\right] \\
W & =\left[\begin{array}{cc}
0.01 & 0 \\
0 & 0.01
\end{array}\right], \quad Z=0.0164 .
\end{aligned}
$$

The goal of this example is to design the robust filter (5) such that the following filtering performance requirements are simultaneously satisfied.

1) The augmented system (18) is mean square bounded.
2) The steady-state error covariance $X_{e e}$ meets

$$
\begin{aligned}
& {\left[X_{e e}\right]_{11} \leq \sigma_{1}^{2}=0.5} \\
& {\left[X_{e e}\right]_{22} \leq \sigma_{2}^{2}=1.2 .}
\end{aligned}
$$

Now, solving the LMIs (42) and (43) for $\varepsilon>0$ and $P_{1}>0$, we obtain

$$
\begin{aligned}
\varepsilon & =0.5168 \\
P_{1} & =\left[\begin{array}{rr}
0.0413 & -0.0006 \\
-0.0006 & 0.0175
\end{array}\right]
\end{aligned}
$$

and subsequently

$$
\begin{aligned}
\Phi & =\left[\begin{array}{ll}
0.0337 & -0.0033 \\
0.0007 & -0.0088
\end{array}\right] \\
\hat{A} & =\left[\begin{array}{ll}
1.2042 & -0.3806 \\
0.0226 & -1.8828
\end{array}\right] \\
\hat{C} & =\left[\begin{array}{ll}
1.4496 & -1.4248
\end{array}\right] \\
\Gamma & =\left[\begin{array}{ll}
40.0196 & 26.1094 \\
26.1094 & 231.1598
\end{array}\right] \\
R & =3.9014, \\
\Theta & =\left[\begin{array}{l}
1.7279 \\
3.5620
\end{array}\right] .
\end{aligned}
$$

Then, solve the QMI (32) to give

$$
P_{2}=\left[\begin{array}{rr}
0.4245 & -0.1443 \\
-0.1443 & 1.1561
\end{array}\right] \text {. }
$$

It is easily seen that $\left[P_{2}\right]_{i i}<\sigma_{i}^{2}(i=1,2)$ and the constraints (22) are satisfied. Next, select the parameter $L$, which meets $\Pi+L L^{T}<0[\Pi$ is defined in (32)], as

$$
L=\left[\begin{array}{l}
0.0800 \\
0.1000
\end{array}\right] \text {. }
$$

Then, for the two cases of $U=1$ and $U=-1$, we obtain the corresponding desired filter parameters from (33)-(34), respectively, as

Case 1:

$$
\begin{aligned}
& U=1 \\
& K=\left[\begin{array}{l}
0.4834 \\
0.9636
\end{array}\right] \\
& G=\left[\begin{array}{rr}
0.5035 & 0.3082 \\
-1.3742 & -0.5098
\end{array}\right]
\end{aligned}
$$

Case 2:

$$
\begin{aligned}
U & =-1 \\
K & =\left[\begin{array}{l}
0.4024 \\
0.8624
\end{array}\right] \\
G & =\left[\begin{array}{rr}
0.6209 & 0.1928 \\
-1.2274 & -0.6541
\end{array}\right] .
\end{aligned}
$$


It is not difficult to test that the prescribed performance objectives are all realized.

\section{CONCLUSIONS}

We have studied the robust filtering problem for uncertain bilinear stochastic discrete-time systems with estimation error variance constraints. Attention has focused on the design of a linear filter, such that for all admissible parameter uncertainties, the error state of the bilinear stochastic system is mean square bounded, and the steady-state variance of the estimation error of each state is not more than the individual prespecified value. We have established both the existence conditions and the explicit expression of desired robust filters in terms of the positive solutions to two quadratic matrix inequalities. A numerical example has been used to show the usefulness of the theory developed.

One of the future research topics would be the multiobjective nonfragile filter design for uncertain bilinear stochastic systems with time delays. That is, the designed filters will be able to tolerate not only the model uncertainty but small changes of the filters themselves as well, while guaranteeing some desired filtering performances. The general framework will be established using the LMI approach in conjunction with regional stability constraints $H_{2}$ and $H_{\infty}$ optimization characterization. In addition, in our opinion, the results obtained in this paper can also be extended to more complex stochastic systems such as sampled-data systems and nonlinear systems. The results will appear in the near future.

\section{ACKNOWLEDGMENT}

The authors would like to thank Prof. F. Yang of Fuzhou University for fruitful discussions. They are also grateful to Prof. H. Unbehauen of Ruhr-University Bochum for detailed comments, to Prof. D. Prätzel-Wolters of the University of Kaiserslautern for helpful suggestions, and to Prof. K. J. Burnham of Coventry University for valuable discussions.

\section{REFERENCES}

[1] R. G. Agniel and E. I. Jury, "Almost sure boundedness of randomly sampled systems," SIAM J. Contr., vol. 9, pp. 372-384, 1971.

[2] D. O. Anderson and J. B. Moore, Optimal Filtering. Englewood Cliffs, NJ: Prentice-Hall, 1979.

[3] E. Beran and K. Grigoriadis, "A combined alternating projections and semidefinite programming algorithm for low-order control design," in Prep. 13th IFAC World Congr., vol. C, San Francisco, CA, 1996, pp. 85-90.

[4] D. S. Bernstein and W. M. Haddad, "Optimal projection equations for discrete-time fixed-order dynamic compensation of linear systems with multiplicative white noise," Int. J. Contr, vol. 46, pp. 65-73, 1987.

[5] C. Bruni, G. DiPillo, and G. Koch, "Bilinear systems: An appealing class of 'nearly linear' systems in theory and applications," IEEE Trans. Automat. Contr., vol. AC-19, pp. 334-348, 1974.
[6] K. J. Burnham, D. J. G. James, and D. N. Shields, "Self-tuning control of bilinear systems," Optim. Contr. Appl. Methods, vol. 8, pp. 147-157, 1987.

[7] F. Carravetta, A. Germani, and M. K. Shuakayev, "A new suboptimal approach to the filtering problem for bilinear stochastic differential systems," SIAM J. Contr. Opt., vol. 38, pp. 1171-1203, 2000.

[8] W. L. DeKoning, "Infinite horizon optimal control of linear discrete time systems with stochastic parameters," Automatica, vol. 18, pp. 443-453, 1982.

[9] —-, "Optimal estimation of linear discrete time systems with stochastic parameters," Automatica, vol. 20, pp. 113-115, 1984.

[10] P. Gahinet, A. Nemirovsky, A. J. Laub, and M. Chilali, LMI Control Toolbox: For Use With Matlab. Natick, MA: Mathworks Inc., 1995.

[11] J. C. Geromel, "Optimal linear filtering under parameter uncertainty," IEEE Trans. Signal Processing, vol. 47, pp. 168-175, Jan. 1999.

[12] J. C. Geromel, P. L. D. Peres, and S. R. Souza, "Output feedback stabilization of uncertain systems through a min/max problem," in Prep. 12th IFAC World Congr., vol. 6, Sydney, Australia, 1993, pp. 35-38.

[13] L. A. Johnston and V. Krishnamurthy, "Finite dimensional smoothers for MAP state estimation of bilinear systems," IEEE Trans. Signal Processing, vol. 47, pp. 2444-2459, Sept. 1999.

[14] L. Li, L. Xie, W.-Y. Yan, and Y. C. Soh, "Design of low-order linearphase IIR filters via orthogonal projection," IEEE Trans. Signal Processing, vol. 47, pp. 434-448, Feb. 1999.

[15] S. O. R. Moheimani, A. V. Savkin, and I. R. Petersen, "Robust filtering, prediction, smoothing, and observability of uncertain systems," IEEE Trans. Circuits Syst. I, vol. 45, pp. 446-457, Apr. 1998.

[16] R. R. Mohler, Bilinear Control Process. New York: Academic, 1973.

[17] R. R. Mohler and W. J. Kolodziej, "An overview of bilinear system theory and applications," IEEE Trans. Syst., Man., Cybern., vol. SMC-10, pp. 683-688, 1980.

[18] A. Saberi, P. Sannuti, and B. M. Chen, $H_{2}$ Optimal Control. London, U.K.: Prentice-Hall, 1995.

[19] R. E. Skelton and T. Iwasaki, "Liapunov and covariance controllers," Int. J. Contr., vol. 57, pp. 519-536, 1993.

[20] R. E. Skelton, S. M. Kherat, and E. Yaz, "Covariance control of discrete stochastic bilinear systems," in Proc. Amer. Contr. Conf., Boston, MA, 1991, pp. 2660-2664.

[21] H. Wang, "Feedback stabilization of bilinear control systems," SIAM J. Contr. Optimiz., vol. 36, pp. 1669-1684, 1998.

[22] Z. Wang and K. J. Burnham, "Discrete-time reduced-order estimator design for bilinear stochastic systems with error covariance assignment," IMA J. Math. Contr. Inform., vol. 18, pp. 99-107, 2001.

[23] Z. Wang and B. Huang, "Robust $H_{2} / H_{\infty}$ filtering for linear systems with error variance constraints," IEEE Trans. Signal Processing, vol. 48, pp. 2463-2467, Aug. 2000.

[24] Z. Wang, B. Huang, and P. Huo, "Sampled-data filtering with error covariance assignment," IEEE Trans. Signal Processing, vol. 49, pp. 666-670, Mar. 2001.

[25] Z. Wang and $\mathrm{H}$. Unbehauen, "Robust $H_{2} / H_{\infty}$-state estimation for systems with error variance constraints: The continuous-time case," IEEE Trans. Automat. Contr., vol. 44, pp. 1061-1065, May 1999.

[26] Z. Wang, J. Zhu, and H. Unbehauen, "Robust filter design with timevarying parameter uncertainty and error variance constraints," Int. J. Contr., vol. 72, pp. 30-38, 1999.

[27] Y. Wang, L. Xie, and C. E. de Souza, "Robust control of a class of uncertain nonlinear systems," Syst. Contr. Lett., vol. 19, pp. 139-149, 1992.

[28] K. Yasuda, S. Kherat, R. E. Skelton, and E. Yaz, "Covariance control and robustness of bilinear systems," in Proc. IEEE Conf. Decision Contr., Honolulu, HI, 1990, pp. 1421-1425.

[29] E. Yaz, "Full and reduced-order observer design for discrete stochastic bilinear systems," IEEE Trans. Automat. Contr., vol. 37, pp. 503-505, Mar. 1992.

[30] E. Yaz and R. E. Skelton, "Continuous and discrete state estimation with error covariance assignment," in Proc. IEEE Conf. Decision Contr., Brighton, U.K., 1991, pp. 3091-3092. 
Zidong Wang (M'94) was born in Gaoyou, Jiangsu, China, on February 21, 1966. He received the B.Sci. degree in mathematics in 1986 from Suzhou University, Suzhou, China, and the M.Sci. degree in applied mathematics in 1990 and the Ph.D. degree in electrical engineering in 1994, both from Nanjing University of Science and Technology, Nanjing, China.

He was appointed as Lecturer at East China Institute of Technology, Nanjing, China, in 1990 and was promoted to be Associate Professor at Nanjing University of Science and Technology in 1994. From January 1997 to December 1998, he was an Alexander von Humboldt research fellow with the Automatic Control Laboratory, Ruhr-University Bochum, Bochum, Germany. From January 1999 to February 2001, he was a Senior Lecturer with the Department of Mathematics, University of Kaiserslautern, Kaiserslautern, Germany. In March 2001, he joined the Control Theory and Applications Centre, School of Mathematical and Information Sciences, Coventry University, Coventry, U.K., as a Senior Staff Member. His research interests include filtering and control for uncertain systems, stochastic systems, nonlinear systems, sampled-data systems, and their applications. He has published more than 60 papers in refereed international journals.

Dr. Wang was awarded the JSPS Research Fellowship in August 1998 from Japan Society for the Promotion of Science. He was a recipient of the Outstanding Science and Technology Development Award from National Education Committee of China (twice in 1996 and once in 1998) and the National Science Investigator Award from the National Natural Science Foundation of China in 1995. He was nominated the outstanding reviewer for the journal Automatica for 2000 and received Standing Membership of the Technical Committee on Control of the International Association of Science and Technology for Development, also in 2000 . He served as a member of program committee for many international conferences, such as the 2001 International Conference on Measurement and Control; the 2001 International Conference on Intelligent Systems and Control; and the 2002 International Conference on Modeling, Identification, and Control.
Hong Qiao (M'00) received the B.Sc. degree in mechanical engineering and control from Xi'an Jiaotong University, Xi' an, China, the M.Phil. degree from Industrial Control Center from Strathclyde University, Glasgow, U.K., and the Ph.D. degree from De Montfort University, Leicester, U.K., in 1996.

From 1995 to 1997, she was a University Research Fellow at De Montfort University. From 1997 to 2000, she was a Research Assistant Professor with City University of Hong Kong. From 2000 to 2001, she was an Assistant Professor with City University of Hong Kong. She is currently a Lecturer with the Computation Department, University of Manchester Institute of Science and Technology, Manchester, U.K., and a Visiting Professor with Fuzhou University, Fuzhou, China. Her interests include information-based strategy investigation, robotics, and control applications. She works in many areas related to neural networks, networks, and data mining. She has successfully applied the concept "attractive regions in the environment" proposed by herself in robotic assembly, robotic grasping, and part recognition. The work has been reported by Advanced Manufacturing Alert. She has more than 25 papers accepted and published in international journals, including IEEE TRANSACTIONS, ASME, and the International Journal of Production Research. She is a paper reviewer for many international top journals, such as IEEE TRANSACTIONS and ASME.

Dr. Qiao was a Member of the Program Committee of the 2001 and 2002 IEEE International Conferences on Robotics and Automation. 